\title{
INTÉRIEURS DOMESTIQUES URBAINS EN FRANCE ET EN ANGLETERRE
}

\author{
Sophie Chevalier \\ Université de Franche-Comté - France
}

Résumé: Cette article concerne une recherche comparative portant sur l'analyse des processus d'appropriation d'objets produits en série dans des ménages de la banlieue parisienne et de Londres. Nous proposons tout d'abord une analyse des décors domestiques français et anglais en dégageant leurs caractéristiques communes et leurs spécificités particulières. Ensuite, nous montrons à travers plusieurs exemples, comment cette construction de l'univers domestique repose sur des processus d'appropriation des objets qui permettent l'expression des identités collectives et individuelles. L'analyse des pratiques et de discours autour des objets domestiques permet de comprendre la façon dont ils peuvent matérialiser différentes dimensions de la vie des ménages, comme les rapports de couple, la structure familiale, l'ancrage géographique, etc. Nous observons alors des contrastes entre Français et Anglais qui ne prennent sens que si on les ramène à un cadre culturel et social spécifique, dans une dynamique historique.

Mots-clés: appropriation, couple, décor domestique, France, Grande-Bretagne, lignée.

Resumo: Este artigo trata de uma pesquisa comparativa baseada na análise dos processos de apropriação de objetos produzidos em série nos lares da periferia parisiense e de Londres. $O$ artigo propõe primeiramente uma análise das decorações domésticas francesas e inglesas investigando suas características comuns e suas especificidades particulares. Em seguida, mostramos através de vários exemplos, como esta construção do universo doméstico repousa sobre os processos de apropriação dos objetos que permitem a expressão das identidades coletivas e individuais. A análise das práticas e dos discursos em torno dos objetos domésticos permite compreender a maneira através do qual eles podem materializar diferentes dimensões da vida e dos lares, como as relações de casais, a estrutura familiar, a inserção geográfica, etc. Nós observamos então os contrastes entre franceses e ingleses que só expressam sentido se inseridos em um quadro cultural e social específico, em uma dinâmica histórica.

Palavras-chave: apropriação, casal, decoração doméstica, França, Grã-Bretanha, linhagem. 
Depuis plusieurs années, je conduis des recherches en milieu urbain sur la culture matérielle et les pratiques de consommation. Elles portent sur la façon dont les individus expriment leur identité individuelle et sociale dans leurs univers domestiques. Plus généralement, je m’intéresse donc aux relations entre sujets et objets au sein de ménages particuliers, qui appartiennent à deux sociétés de consommation de masse, la France et l'Angleterre. ${ }^{1}$ Une première enquête de terrain ${ }^{2}$ a été menée dans des tours de logements sociaux de la banlieue ouest de Paris (commune de Nanterre, quartier des Fontenelles). Plus précisément, l'enquête concerne soixante ménages, locataires de logements identiques, avec des espaces contraints. Leurs résidents sont des ouvriers ou des employés. La deuxième enquête porte sur un lotissement - Jersey Farm - situé sur la commune de St Albans, au nord de Londres. Les maisons sont de tailles variées, allant d'une chambre à coucher pour les bungalows à quatre chambres pour les grandes maisons. ${ }^{3}$ Les occupants sont propriétaires; pour la plupart ils travaillent comme employés dans le secteur tertiaire et quelques-uns sont des ouvriers à la retraite. J'ai recontré trente ménages.

Le milieu socio-économique auquel appartiennent ces informateurs est semblable, j'ai postulé qu'il est comparable (pour la discussion voir Chevalier, 1996, p. 116). Pour qualifier "grossièrement" celui-ci, nous dirons qu'il s'agit d'un groupe appartenant à une classe moyenne "inférieure”. Elle est souvent stigmatisée par une certaine sociologie comme étant la proie idéale de la consommation de masse capitaliste. Cependant, la thèse selon laquelle le système économique des sociétés occidentales conduirait à une homogénéisation culturelle, peut être infirmée par des analyses portant sur ces groupes sociaux comme le montrent depuis quelques années les études anthropologiques sur la "globalisation".

Les généralisations faites dans cet article ne peuvent donc s'étendre qu'à ce groupe social, néanmoins celui-ci comprend une grande part de la population des deux sociétés considérées. Cependant, comme toute comparaison, ces

\footnotetext{
1 J'ai commencé récemment de nouvelles recherches sur une société post-communiste, la Bulgarie. Elles s’orientent plus vers une analyse des stratégies économiques de survie des ménages domestique.

2 L’enquête a été menée entre 1988 et 1990; le terrain anglais entre 1994 et 1995.

3 Les maisons sont soit en "terrasse": série de plusieurs maisons accolées les unes aux autres; soit ce sont des maisons “semi-détachées”: maisons accolées deux par deux, dites “jumelles” en français; ou encore des maisons “détachées”: maisons séparées des autres.
} 
différences renvoient à un autre niveau culturel et historique, à des aspects fondamentaux de ces sociétés, comme la structure de parenté, par exemple.

Mon étude s'articule autour de l'opposition dialectique entre l'individuel et le collectif que je pense aussi fondamentale que celle qui oppose l'économique au culturel. L'analyse des pratiques de consommation domestique permet de comprendre l’intégration et la résolution de ces dilemmes entre les différentes dimensions de la vie sociale. La construction des univers privés à travers les pratiques de consommation peut être considérée comme les interprétations subjectives faites par les acteurs de leurs expériences objectives de la réalité sociale et économique. Cette élaboration se fait à l'articulation entre l'individuel et le collectif, c'est-à-dire entre le désir d'expression de soi et la nécessité de signifier son appartenance à un groupe. Ce processus tente alors de concilier les choix individuels et les contraintes sociales.

\section{Des espace domestiques différents}

Le séjour, ${ }^{4}$ est la pièce à laquelle je m'intéresse particulièrement, lieu d'intersection entre le privé et le public. Elle ne constitue pas seulement l'espace de présentation de soi à travers meubles et objets, mais aussi celui où les relations familiales sont objectivées face au visiteur extérieur, dans une image idéalisée du foyer. Il est important de remarquer ici que les notions de privé et de public n'ont pas les mêmes significations en France et en Angleterre. ${ }^{5}$

Les objets qui composent le décor des ménages français et anglais " $n$ 'ont rien de spécial”, selon l'expression d'un informateur, car la plupart d'entre eux ont été produits en grande série. En eux-mêmes, ils ne singularisent pas l'intérieur qu'ils meublent. En revanche, c'est la combinaison des meubles et objets, les relations qu'ils entretiennent entre eux, à chaque fois unique, qui sont l'expression de l'identité du ménage, bien que certaines configurations soient communes à tous les ménages.

Dans chaque séjour français, on trouve un certain nombre de meubles quelque soit la taille de la pièce, qui sont: (1) table/chaises/buffet et (2)canapé/ (fauteuils)/table basse plus télévision. Le discours des informateurs confirment

\footnotetext{
4 J'utilise le terme de "séjour" comme la plupart de mes informateurs français; les Anglais parlent de "lounge".

5 Je n’ai pas la place ici d'explorer cette intéressante différence.
} 
les observations du chercheur: ils mettent en avant ces deux groupes mobiliers. De petites différences apparaissent liées à la classe d’âge: les jeunes ménages accordent surtout de l'importance au deuxième groupe. L'ensemble "salle à manger” disparaît, sauf le buffet ou l'un de ses “descendants” de style moderne qui subsiste.

L'ameublement anglais, qui diffère du français, est le "three-piece suite”. Cet ensemble mobilier se compose d'un canapé et de deux fauteuils assortis disposé, traditionnellement, autour de la cheminée (et/ou du téléviseur). Les maisons récentes n'ont souvent pas de cheminée: les habitants en font installer une dans laquelle brûle un "faux" feu, à gaz ou à électricité. Ceux qui ne veulent pas dépenser une telle somme d' argent dispose à la place un buffet-bibliothèque nommé “unit”. Ce meuble, comme le dessus de la cheminée, est utilisé pour exposer des objets décoratifs et des photographies. Les informateurs insistent sur la nécessité d'avoir un point de convergence des regards dans leur pièce, comme une cheminée. En fait, le téléviseur entre souvent en compétition avec celle-ci dans l'organisation de l'espace, ce que mes interlocuteurs reconnaissent volontiers et le plus souvent déplorent. L'ensemble “salle à manger” n’appartient pas au mobilier de "base" du séjour; il est rarement central dans le séjour, qui peut contenir parfois un buffet ou un dressoir dit "Welsh dresser".

L'analyse formel des décors des séjours montre que l'on rencontre toujours et dans les deux pays une configuration, ou système mobilier, à chaque fois particulière, avec une “base normée”; c'est-à-dire quelques meubles qui semblent essentiels et nécessaires aux individus, différents d'ailleurs pour les Français et les Anglais. Le mobilier de "base" caractérise la pièce et matérialise le "foyer" (ou "home”). Mais ce foyer s'inscrit dans un cadre culturel spécifique: des objets ou des meubles plus ou moins similaires sont disposés dans le séjour pour satisfaire à des exigences culturelles d'organisation de l'espace. Ainsi il existe deux façons formelles d'incarner ce que doit être un "foyer".

Cependant, cette élaboration peut aussi être considérée comme un processus qui conduit à transformer des objets de consommation de masse bien souvent similaires dans leur forme et leur fonction - en objets inaliénables. C'est-à-dire des objets qui permettent la construction et l'expression d'une identité collective, comme je viens de le démontrer, mais aussi individuelle. Ce processus peut être décrit comme une appropriation (Miller, 1987). Les individus mettent en oeuvre des processus de re-socialisation de l'environnement matériel, en réponse à une possible expérience d'aliénation. Il convient alors de 
comprendre comment des consommateurs personnalisent à travers leur mode de vie des objets qui sont le plus souvent des "marchandises" produites en grande série.

\section{L'individu, le couple et la famille dans l'espace domestique}

Les relations entre les membres de la famille, en particulier entre les conjoints, sont matérialisées dans l'espace du séjour. La disposition des meubles dans l'espace et ses transformations offrent des indices sur des changements éventuels dans les rapports familiaux dans l'univers domestique.

En Angleterre, la cheminée a toujours été le point de convergence du séjour avec des sièges disposés en demi-cercle autour. De nos jours, la diffusion du chauffage central et l'introduction de la télévision ont entraîné des modificiations, mais d'un degré moindre au regard des ménages français. Car cette disposition contient toujours la possibilité d'avoir des activités individuelles en co-présence, et n'implique pas de hiérarchie à priori entre les individus. En France, l'importance traditionnellement accordée à l'ensemble salle à manger, ainsi que la disposition des meubles, créent un espace fermé sur lui-même: autour de la table, on se fait face, comme d'ailleurs, dans la partie du séjour où les sièges sont disposés de manière à ce que les résidents se regardent les uns les autres. Cette organisation de l'espace, en particulier, la famille assise autour de la table de salle à manger, peut avoir des implications sur les rôles homme/ femme. Elle impose aussi un certain formalisme dans les comportements ainsi qu'une hiérarchie entre les membres de la famille.

Cette différenciation des rôles masculins et féminins trouve à s'exprimer dans la façon dont les relations entre sexes sont matérialisés dans l'espace du séjour. Prenons comme exemple M. et Mme Probst: ils sont mariés avec deux grands enfants, dont l'un vit encore avec eux. Ils ont dans la cinquantaine. M. Probst est employé de bureau dans une grande entreprise (Kodak) et Mme Probst est au foyer. Tous les deux sont originaires de la banlieue parisienne. Ils ont acheté tout leur ameublement en 1956 qui constitue donc un ensemble mobilier homogène de style "années 50". Les seuls éléments ajoutés sont des tables gigognes et les deux canapés en cuir brun. Tous les meubles sont chargés d'objets: il n'est pas concevable, esthétiquement, de laisser des tables vides, qui n'ont pas ou peu de fonctions utilitaires. La pièce est divisée en deux parties: salle à manger, et séjour. La première partie contient les objets décoratifs les 
plus "précieux" qui sont mis en valeur sur le buffet. Ils sont en affinité fonctionnelle avec l'usage de cette espace, ainsi salle à manger/assiettes par exemple, comme cette assiette qui proviendrait du service de Limoges de l’Elysée. ${ }^{6}$ La plupart de ces objets ont été hérités du côté maternelle par Mme Probst, ils évoquent la famille et l'origine. L'autre partie du séjour, quant à elle, est plus moderne avec ces canapés en cuir et ces lithographies abstraites, cadeaux professionnels offerts à M.Probst par ses clients. Les objets exposés sont des cadeaux ou souvenirs de voyages: ils évoquent l'univers extérieur et le monde professionnel.

Cette partition du séjour apparaît comme une différenciation sexuelle, avec un espace féminin évoquant et un espace masculin, en accord avec les rôles traditionnels attribués à chaque sexe (Bourdieu, 1980; Humphrey, 1974). Ces deux parties sont reliées entre elles par la bibliothèque, transition entre le féminin et le masculin, sur laquelle sont exposés des photographies des enfants et petitsenfants, témoignage de la continuité familiale. Dans son discours, Mme Probst confirme ces observations, mais il apparaît que c'est elle qui a élaboré cette construction particulière. A Jersey Farm, c'est le couple en tant que tel qui est matérialisé dans le séjour: je n'ai jamais observé une “sexualisation” de l'espace comme chez les Probst. Cet absence peut être liée au rôle secondaire joué par la partie salle à manger dans les séjours anglais, et s'exprime à travers la disposition spatiale de l'ameublement dans le séjour.

Cependant, l'organisation de l'espace domestique français subit une transformation historique, qui reflète les changements des rapports au sein du ménage (de Singly, 1998). Aujourd’hui, la place dominante de la télévision, qui a souvent comme conséquence la disparition ou la marginalisation spatiale de la partie salle à manger dans le séjour, introduit de nouvelles façons d'être ensemble en couple ou en famille. Ce n'est pas seulement l'organisation de la pièce en tant que telle qui s'est trouve transformée, mais elle exprime aussi une plus grande individualisation des activités en coprésence (de Singly, 1996) et une disparition progressive des contraintes identitaires traditionnelles liées au sexe de l’individu, visibles dans la répartition des tâches.

\footnotetext{
${ }^{6}$ L’Elysée est la résidence du Président de la République français; il semblerait que chaque nouveau président puisse commander un nouveau service de porcelaine à une des grandes manufactures nationales, par exemple Limoges.
}

Horizontes Antropológicos, Porto Alegre, ano 6, n. 13, p. 113-125, jun. 2000 
Tant en France qu'en Angleterre, les relations entre sexes sont devenues plus égalitaires. La répartition sexuée des espaces dans la sphère domestique, observable chez les Probst, et la spécialisation féminine/masculine des activités tendent à se réduire. L'analyse de la façon dont les relations entre sexes sont matérialisées dans l'univers privé permet d’observer la continuité et les transformation dans les modèles culturels, avec le constat que les modes de vie des jeunes couples des Fontenelles et de Jersey Farm deviennent très similaires.

Pourtant ces changements doivent être relativisés. Quand je faisais mes entretiens, les ménages anglais insistaient généralement pour me rencontrer en tant que couple et me fixaient un rendez-vous à un moment où mari et femme étaient disponibles. Quant aux résidents des Fontenelles, ils me suggéraient de discuter avec la femme, et si le mari était présent, il restait très souvent silencieux.

Les différences observées entre Les Fontenelles et Jersey Farm dans la matérialisation des rapports dans le couple et dans famille, trouvent un prolongements dans l'analyse des liens qui se contruissent, grâce aux objets, entre le monde privé et l'extérieur.

\section{Les objets comme liens avec le monde extérieur}

On peut considérer que la relation entre l'individu et le groupe est médiatisée par le monde des objets. Le discours des informateurs autour de leur décor met en scène cette médiation, par un lien qui repose sur la réalité ou qui est une construction discursive. Dès ce moment, ces objets vont “matérialiser” un ou des individus, un événement ou un lieu, par exemple. L'énonciation de ce lien, reposant sur leurs présences dans le séjour, équivaut à reconnaître qu'ils participent à l'expression de l'identité du ménage, par ce qu'ils matérialisent. Ils deviennent des supports pour des discours, en particulier dans un processus de remémoration qui permet l'évocation d'un récit de vie.

Ainsi si le décor pris dans son ensemble incarne l’idée de foyer, les objets qui participent à cette construction matérialisent différentes dimensions de cette identité. L'expression de celle-ci est personnelle, mais le choix de certaines médiations plutôt que d'autres est en revanche culturelle.

Il est nécessaire de préciser que l'aspect formel d’un objet ne joue pas un rôle important dans la matérialisation: deux objets similaires peuvent matérialiser des événements très divers, par exemple. En revanche, c'est plutôt le mode de circulation, la provenance, de ces objets qui peut entraîner des contraintes dans 
les choix individuels. Une analyse sociologique de la circulation des objets permet de comprendre comment s'élabore cette articulation entre individu et collectif (Chevalier, 1998).

Les objets hérités et les cadeaux sont plus contraignants que des objets achetés: ils participent à une mise en scène de soi à travers l'exposition de l'ensemble des relations sociales que nous entretenons, tant avec les vivants qu'avec les morts. S'approprier un héritage ou un présent est reconnaître son rapport aux autres, et accepter d'entretenir des liens avec eux. Ces objets matérialisent leurs donateurs et les événements et/ou lieux liés à ces transferts, qui peuvent être différents moments du cycle de vie - sauf pour l'héritage qui est lié au décès d'un ascendant - , diverses personnes, parents, amis ou voisins, et des lieux variés, lointains ou proches.

En revanche, l'achat se caractérise par une absence de contraintes sociales directes, puisqu'ils ne médiatisent pas de liens concrets. Ce dernier s'inscrit dans la "liberté marchande", rendu possible par l'usage de la monnaie (Simmel, 1987): c'est-à-dire qu'acheter une marchandise permet de se libérer des liens sociaux, de pouvoir sortir d'un rapport social sans contraintes. Lors d'un achat fait dans un grand magasin, dans le cadre un échange impersonnel, le propriétaire de cette acquisition est libre de construire des relations imaginaires entre cette marchandise et une personne de son choix. L'objet hérité ou offert, quant à lui, se situe dès le départ dans le lien social, il s'inscrit dans une dynamique des échanges entre individus. Il est donc moins malléable que celui acheté.

Dans les intérieurs domestiques, la provenance des pièces mobilières qui les composent est variée: c'est l'articulation d'objets de différentes origines qui permet l'expression des dimensions de l'identité, tant collectives qu'individuelles. Rares sont les univers domestiques, pris en compte lors de mes enquêtes, complètement vides d'objets reçus ou hérités, et élaborés qu'avec des objets achetés: il subsiste toujours au moins quelque chose qui a été offert. Dans le cas contraire, la personne est décrite par mes informateurs comme vivant hors de tous liens sociaux, comme quelqu'un dont l'identité se serait construite isolément et n'exprimerait qu'elle-même. A l'autre extrême, un univers privé constitué que de cadeaux ou d'héritages ne laisse pas de place à l'expression individuelle. Mrs Devlin décrit la maison de son enfance: "Les objets décoratifs de mes parents étaient presque tous des cadeaux, pas vraiment leur choix. Moi, j'ai décidé d'acheter la plupart de mes objets pour avoir le choix”. Derrière tous ces objets, la présence des autres est écrasante. Cette revendication 
individualiste peut être considérée comme un phénomène de génération: ceux qui nous ont précédés, ont bien souvent vécus dans des décors qui avaient été constitués par d'autres sans ressentir la nécessité de les changer au nom de l'expression de soi.

Ainsi tout décor est une articulation entre individuel et collectif. Comme anthropologue, c'est le contenu de cette identité collective, matérialisée dans des objets, qui m’intéresse en premier. Certaines différences sont apparues entre nos informateurs français et anglais comme je vais le montrer à travers plusieurs exemples, extraits de mes entretiens.

Français comme Anglais reçoivent en cadeaux de mariage des objets pour leur intérieur comme de la vaisselle - surtout des verres en cristal -9 qui sont enfermés dans le buffet s’il existe. De plus, à Jersey Farm, il est courant de recevoir du mobilier en cadeau.

Robby et Janet Moore, trente-et-un ans tous les deux, viennent d'emménager dans ce qui est leur première maison en commun. Leur mobilier et leurs objets sont constitués presque entièrement de présents: le meuble principal, un buffet-bibliothèque est un cadeau de mariage de la mère de Janet. D'après leurs propres termes, c'est l'élément central de la pièce, le point de convergence des regards. Ils y ont disposé de nombreux bibelots: entre autres, des verres en cristal et une coupe, cadeaux de fiançailles de leurs parents. Une photographie est placée au-dessus du buffet: il s'agit de celle de l'église locale où leur cérémonie de mariage s'est déroulée. C’est un cadeau de mariage de la parenté comme les deux photographies, l'une des fiançailles et l'autre de leur mariage, accrochées aux murs. Dans ce séjour, l'accent est mis sur leur couple et les rites de passage qui ont conduit à sa constitution sociale. Mes informateurs anglais sont plus enclins à mettre en valeur des cadeaux de fiançailles et de mariage. Dans les ménages français de la même classe d'âge, les fiançailles, si elles existent, ne sont que très rarement évoquées à travers des objets. Ils exposent peu de photographies de leurs mariages, nombreuses à Jersey Farm. Les présents reçus au mariage sont disposés dans le séjour, mais on ne retrouve pas la même valorisation que chez les jeunes couples anglais.

Ce constat rejoint celui que j'avais fait dans mon analyse comparée des projets de transmission des ménages du lotissement de Saint-Albans et de ceux de Nanterre (Chevalier, 1996). Les ménages français poursuivent un dessein de transmission au sein de leur lignée; bien souvent double, puisqu'il concerne à la fois le contenu de leur appartement de banlieue et la maison de famille, $\mathrm{y}$ 
compris son décor, en province. A travers la façon dont ils appréhendent la culture matérielle, ils mettent en avant la postérité. Tandis que le logement et son aménagement sont d'abord un projet du couple en Grande-Bretagne, car ils ne s'inscrivent pas dans une dimension de la lignée, ils sont l'incarnation de l'alliance. L'importance de celle-ci s'exprime à travers l'exposition d'objets qui ont été offerts et qui matérialisent un événement, un rite de passage, moment fondateur d'un nouveau ménage.

Les parents de Janet et Robby leur ont donné tous les éléments qui font qu'un couple anglais puisse se sentir dans un vrai foyer (“a real home”). Si les parents des jeunes couples français ne participent pas à l'installation de leurs enfants dans un appartement des Fontenelles, on peut faire l'hypothèse que ce logement ne peut probablement pas constituer à leurs yeux un "vrai” foyer. Ils aideront peut-être leurs enfants à aménager dans un autre lieu, plus valorisé, mais toujours avec l'idée que c'est la maison d'origine, le plus souvent en province qui est le "vrai foyer" familial.

L’origine est fortement mise en valeur dans les intérieurs des Fontenelles, par des présents qui les lient non seulement à leur famille, mais aussi à leur lieu d'origine. M. et Mme. Rufin, tous les deux dans la trentaine, sont mariés avec deux enfants. M.Rufin est dans la fonction publique, Mme Rufin est au foyer. Ils sont nés dans La Creuse, une région rurale du centre de la France, où leurs familles vivent. Ils possèdent une seconde résidence là-bas, qu'ils utilisent comme maison de vacances. Dans leur appartement de banlieue, les objets décoratifs ont été souvent achetés dans des brocantes ou sont des souvenirs de vacances. Mme Rufin a reçu une petite pendule de ses collègues quand elle a quitté son emploi après la naissance de son premier enfant. Une photographie de son père décédé est disposé sur le buffet. Les assiettes à poisson en faïence de Quimper (Bretagne) sont des cadeaux de mariage. Cependant, l'élément remarquable de leur décoration est aux murs. Le couple y a accroché des oiseaux empaillés, recueillis par des chasseurs dans La Creuse (parfois trouvés au bord de la route). Ces animaux sont donnés à empailler à un taxidermiste. M. et Mme Rufin sont aussi amateurs de tapisseries, car Aubusson, qui est une manufacture de tapisserie très connue, est situé près de leur ville natale. Ainsi ils en ont acquises plusieurs pour décorer leur appartement. M. Coderc-Deschamps, un jeune homme de l'Ariège, ${ }^{7}$ a aussi suspendu aux murs un animal empaillé audessus de son canapé, une tête de sanglier, une trophée de chasse offerte par un de ses parents. En revanche, les Anglais de Saint-Albans disposent de 
nombreux objets de décoration murale qui font référence au lieu où ils habitent, par exemple, la ville de Saint-Albans, même si seul un tiers des ménages a l'un ou l'autre des conjoints originaires de cette commune. Comme Mrs Roberts, originaire des Midlands qui décrit la gravure qu'elle a mis aux murs: “C'est le château local, celui de Saint Albans, que nous avons acheté quand nous sommes arrivés ici”.

On pourrait dire que les uns se soucient surtout de savoir “où ils vont” à travers la succession des maisons qu'ils ont occupés ou qu'ils occuperont; alors que les autres, les Français, tiennent à se souvenir “d'où ils viennent”. Certaines différences montrent que les Français mettent en valeur leur groupe d'appartenance, leur lignée et leur lieu d'origine, et que les Anglais vont donner de l'importance à leur couple, à leur réseau amical et à leur lieu de vie et de travail. D’une manière générale, ces contrastes sont autant de manières culturelles de résoudre le dilemme entre la nécessité d'une certaine stabilité et la réalité du mouvement, dimensions inhérentes à la vie contemporaine urbaine.

\section{Conclusion}

Mes analyses (Chevalier 1994, 1995, 1996, 1998) montrent que, d'une part, mes informateurs élaborent des univers tout à fait personnalisés avec des objets de série; et que, d'autre part, d'importantes différences culturelles subsistent (Chevalier, 1996). Les significations que j’ai pu attribuées à ces contrastes entre les résidents de St-Albans et des Fontenelles ne prennent sens que si on les ramène à un cadre culturel et social spécifique. Ainsi il semblerait que la différenciation sexuelle des rôles, la continuité d'une lignée familiale et les origines géographiques s'expriment moins à travers la culture matérielle des intérieurs des classes moyennes anglaises que dans ceux des Français. En revanche, les premiers matérialisent leur couple et leurs liens avec leur lieu de résidence avec plus de vigueur que les habitants des Fontenelles. Ces constatations ont des racines historiques qui nous ramènent à l'histoire de l'urbanisation et des transformations des structures familiales, toujours en train de se faire.

${ }^{7}$ Département très rural du sud-est de la France. 
Cependant, le plus étonnant n'est peut-être pas qu'il existe des contrastes entre Anglais et Français, mais qu'ils perdurent de nos jours et dans ce groupe social, soumis soit-disant à une uniformisation culturelle due au système capitalisme. Les considérations économiques ne définissent jamais complètement les dimensions culturelles et sociales des pratiques de consommation.

\section{Références}

APPADURAI, Arjun (Ed.). The social life of things: commodities in cultural perspective. Cambridge: Cambridge University Press, 1986.

BAUDRILLARD, Jean. Le système des objets: la consommation des signes. Paris: Gallimard, 1968.

BOURDIEU, Pierre. La maison ou le monde renversé. In: BOURDIEU, Pierre. Le sens pratique. Paris: Editions de Minuit, 1980. p. 441-461.

CARRIER, James. Reconciling commodities and personal relations in industrial society. Theory and Society, 19, p. 579-598, 1990.

CHEVALIER, Sophie. L'ameublement et le décor intérieur dans un milieu populaire urbain: approche ethnographique d'une vraie-fausse banalité. Thèse (Doctorat)-Université de Paris X-Nanterre, Paris, 1992.

CHEVALIER, Sophie. Au-delà d'une apparente banalité et d'un standard: des décors domestiques particuliers. Archives Suisses des Traditions Populaires, 90, p. 165-185, 1994.

CHEVALIER, Sophie. The Anthropology of an apparent banality: a comparative study. Cambridge Anthropology, 18, 3, p. 25-39, 1995.

CHEVALIER, Sophie. Transmettre son mobilier?. Ethnologie Française, v. 26, n. 1, p. 115-128, 1996.

DOUGLAS, Mary; ISHERWOOD, Baron. The World of Goods. New York: Basic, 1979.

HUMPHREY, Caroline. Inside a Mongolian tent. New Society, 1974. 
McCRACKEN, Grant. Culture and consumption: new approaches to the symbolic character of consumer goods and activities. Indiana: Indiana University Press, 1988.

MILLER, Daniel. Material culture and mass consumption. Oxford: Blackwell, 1987.

MILLER, Daniel. Appropriating the State on the Council Estate. Man, n. 23, p. 353-372, 1988.

MILLER, Daniel. Capitalism: an ethnographic approach. Oxford: Berg, 1994.

MORLEY, Christine. The three-piece suite: the survival of a popular form, its critics and consumers. Middlesex University, Master of History of Design, 1990.

PUTNAM, Tim; NEWTON, Charles (Ed.). Household's choices. London: Middlesex Polytechnic, 1990.

SAHLINS, Marshall. Age de pierre, âge d'abondance. Paris: Gallimard, 1976. SIMMEL, Georg. Philosophie de l'argent. Paris: PUF, 1987.

SINGLY, François de. Le soi, le couple et la famille. Paris: Nathan, 1996.

SINGLY, François de. Habitat et relations familiales. Paris: PUCA, 1998. 